$\begin{array}{llllllllllllllll}\text { A C T A } & \text { C H E M I C A S C A N D I N A V I C A } & 10 & (1956) & 447-450\end{array}$

\title{
The Change in Reduced Diphosphopyridine Nucleotide (DPNH) Fluorescence upon Combination with Liver
} Alcohol Dehydrogenase (ADH)

\author{
P. D. BOYER and HUGO THEORELL
}

Medicinska Nobelinstitutet, Biokemiska avdelningen, Stockholm, Sweden

\begin{abstract}
Measurement of the intensity of fluorescence at different wave lengths has shown that the fluorescence of DPNH is shifted from a maximum at about 450 millimicrons to about 415 millimicrons and increased in intensity upon combination of the DPNH with liver alcohol dehydrogenase.
\end{abstract}

\begin{abstract}
Drevious communications from this laboratory have described a fluorescence I recorder and its application to the study of the combination of riboflavin phosphate with the old yellow enzyme and the velocity of the formation or disappearance of DPNH ${ }^{1-3}$. The measurements had shown that DPNH gave the same intense fluorescence whether it was free in solution or combined with liver alcohol dehydrogenase (ADH). In view of the shift in absorption spectra shown by DPNH when it is bound to $\mathrm{ADH}^{4}$ together with the known spectral shifts of many dyes upon binding to proteins, it seemed possible that DPNH might show some change in the intensity of fluorescent light emitted at different wavelengths upon binding to ADH. Simple modifications of the apparatus previously used allowed test of this possibility. The results obtained are given in this paper. They show that the binding of DPNH by ADH results in a definite lowering of the wavelength for the fluorescence maximum and an increase in the intensity of the fluorescence.
\end{abstract}

\section{EXPERIMENTAL}

The apparatus previously described ${ }^{1}$ was modified by removing the exit filter and placing before the phototube a suitable, covered compartment for insertion of various interference filters in the exit beam.

The ADH was prepared essentially as described by Bonnichsen 5, with final crystallization from dilute phosphate buffer at pH 7. The colorless preparation used was recrystallized twice, and was approximately $70 \%$ pure based on activity tests assuming that

Acta Chem. Scand. 10 (1956) No. 3 


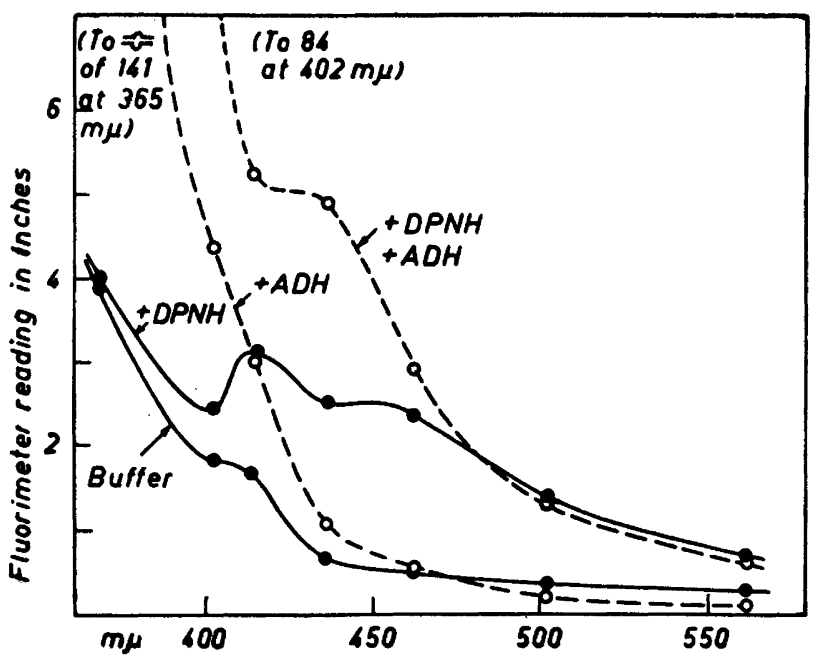

Fig. 1. Observed fluorimeter readings at different wavelengths. [DPNH] at $4 \times 10^{-6} M$ $[A D H]$ at approximately $4 \times 10^{-6} M$, in $0.09 M$ phosphate buffer, $p H 7.0,0.01 M$ semicarbazide and $0.03 \mathrm{M}$ ethanol. Inlet slit at $7 \mathrm{~mm}$, exist slit at $6 \mathrm{~mm}$, and potentiometer sensitivity at 0.1 volt per 10 inches. (Expt. F-13).

all the material absorbing at $280 \mathrm{~m} \mu$ was ADH *. The DPNH was a preparation of Sigma Chemical Company with a ratio of absorption at $260 \mathrm{~m} \mu$ to that at $340 \mathrm{~m} \mu$ of 2.59 . It was over $98 \%$ reduced as measured by treatment with ADH and ethanol in the presence of semicarbazide, and the absorption at $340 \mathrm{~m} \mu$ was nearly completely abolished by treatment with ADH and acetaldehyde.

In the experiments reported herein the DPNH was dissolved in $2.65 \mathrm{ml}$ of $0.1 \mathrm{M}$ phosphate buffer, $\mathrm{pH} 7.0,0.3 \mathrm{ml}$ of $0.1 \mathrm{M}$ semicarbazide $\mathrm{pH} 8$, and $0.05 \mathrm{ml}$ of 1:10 ethanol. A small catalytic amount of ADH was added and the solutions allowed to stand for one hour to reduce the final traces of DPN. This insured that any observed shifts in fluorescence in later experiments were not the result of reduction of DPN.

Comparisons of fluorescence were made with the same buffer mixture as that used for dissolving the DPNH, with addition of appropriate small volumes of ADH crystal suspension or solution in approximately $0.01 \mathrm{M}$ phosphate buffer, pH 7.0.

\section{RESULTS AND DISCUSSION}

In Fig. 1 are shown the observed fluorimeter readings at different wavelengths for the buffer mixture, buffer $+\mathrm{DPNH}$, and buffer $+\mathrm{ADH}$, and buffer + DPNH and ADH. The amount of ADH was in excess of that of the DPNH as ADH contains two active combining sites per mole 4 .

In Fig. 2 are shown the fluorescence increments for free and ADH-bound DPNH.

The data show clearly that the combination of the DPNH with ADH results in a rather pronounced increase in fluorescence intensity together with a deorease in the wave-length for the maximum observed fluorescence. This shift in observed fluorescence likely results from changes in the properties of the 


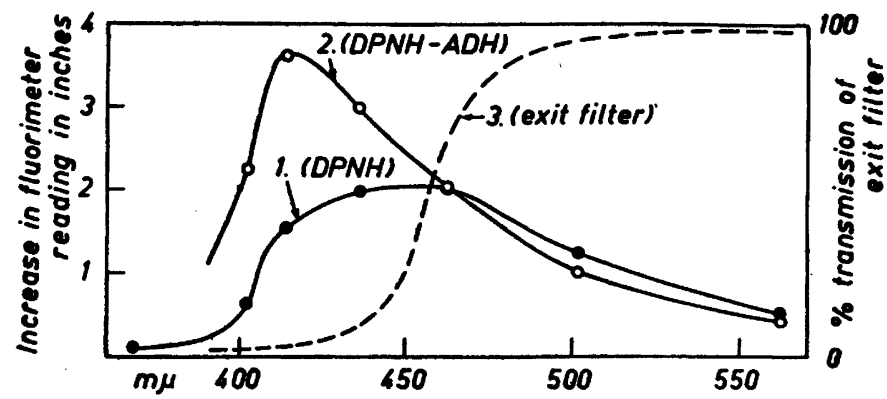

Fig. 2. Increase in fluorimeter reading at different wavelengths for DPNH (1) and $D P N H-A D H(2)$, corrected for the fluorescence of $A D H$. The points represent the averages of these experimental series under conditions as for Fig. 1. Also shown is the per cent trans. mission of the exit filter used in the apparatus as previously described (3).

DPNH molecule upon binding, but the possible formation of a new fluorescent grouping by the combination of $\mathrm{DPNH}$ with $\mathrm{ADH}$ also merits consideration.

Previously it was reported that combination of ADH with DPNH did not result in any change in the measured fluorescence ${ }^{6}$. This observation was entirely valid under the experimental conditions used in those experiments. The exit filter of the original apparatus was chosen primarily for measurement of flavin fluorescence. As noted in Fig. 2, the light transmission by this exit filter was fortuitously of such characteristics as to completely mask the change in the DPNH fluorescence.

The observed fluorescence increase as given in Fig. 1 may be corrected for the known sensitivity of the photomultiplier tube used (RCA IP21) to give a closer approximation of the actual relative intensity of the emitted fluorescence. Such corrections only increased the observed maximum of the DPNH fluorescence by about $5 \mathrm{~m} \mu$ and did not change that of the ADH-bound DPNH.

Titrations of the approximate combining capacity of ADH for DPNH based on the fluorimetric shift gave values in the range expected from the activity and the absorption at $280 \mathrm{~m} \mu$ of the ADH. However, further instrumental modifications are necessary for accurate measurement of combining proportions based on fluorescence changes.

Possible instrumental modifications which would facilitate more accurate measurements of the fluorescence shift and use of the shift for stoichiometric and kinetic measurements should be mentioned. A particularly troublesome difficulty was the high fluorescence given by ADH in the lower wavelength region (see Fig. 1). In part this difficulty may be decreased by use of exciting light such that none of the light can pass through the exit filter and thus reach the photocell by reflection. The inlet filter of the present instrument allows some light in the $400-430 \mathrm{~m} \mu$ region to pass and this results in variations in fluorescence due to the presence of dust particles in the solutions used or small amounts of turbidity in the ADH preparations. Also by the judicious selection of filters or use of suitable monochromators it may be possible to 
decrease the fluorescence of the ADH by eliminating the lower wavelengths of light which brings about the fluorescence of ADH.

A definite limitation in the use of filters and monochromators is the decreased sensitivity of the measurements. For example, the data reported in Figs. 1 and 2 were obtained employing the maximum sensitivity of the instrument.

The results with the liver alcohol dehydrogenase suggest further experiments, preferably with improved experimental conditions, to find if the combination of DPNH with other dehydrogenases will result in fluorescence shifts or if the shift is limited to liver alcohol dehydrogenase. Also, other fluorescent coenzymes and substrates may show shifts in fluorescence upon combination with their respective apoenzymes. Measurement of such fluorescent shifts may become a valuable experimental tool.

Mr. B. Hellström and Mr. L. Salomonsson gave valuable assistance in modification of the apparatus. One of us (P.D.B.) is indebted to a grant from the Guggenheim Foundation which made this work possible.

\section{REFERENCES}

1. Theorell, H. and Nygaard, A. P. Acta Chem. Scand. 8 (1954) 877.

2. Theorell, H. and Nygard, A. P. Acta Chem. Scand. 8 (1954) 1649.

3. Theorell, H., Nygaard, A. P. and Bonnichsen, R. Acta Chem. Scand. 8 (1954) 1490.

4. Theorell, H. and Bonnichsen, R. Acta Chem. Scand. 5 (1951) 1105.

5. Bonnichsen, R. Acta Chem. Scand. 4 (1950) 715.

6. Theorell, H., Nygaard, A. P. and Bonnichsen, R. Acta Chem. Scand. 9 (1955) 1148.

Received December 30, 1955. 